\title{
HYBRID REPRESENTATIONS FOR AUDIOPHONIC SIGNAL ENCODING
}

\author{
L. DAUDET AND B. TORRÉSANI
}

\begin{abstract}
We discuss in this paper a new approach for signal models in the context of audio signal encoding. The method is based upon hybrid models featuring transient, tonal and stochastic components in the signal. Contrary to several existing approaches, our method does not rely on any prior segmentation of the signal. The three components are estimated and encoded using a strategy very much in the spirit of transform coding. While the details of the method described here are taylored to audio signals, the general strategy should also apply to other types of signals exhibiting significantly different features, for example images.
\end{abstract}

\section{INTRODUCTION}

A large number of modern signal coding strategies are based on approaches that combine transform coding with suitable quantization and entropy coding of the corresponding coefficients. The transform step is generally achieved via an expansion with respect to a suitably chosen basis, i.e. a basis of some underlying space of signals, with respect to which signals in the considered class are expected to have a "short" expansion. Such approaches have been especially successful for still image compression, for which it has been shown that wavelet (or sub-band) expansions are extremely well adapted, and allow one to achieve high compression rates. This seems to be due to the very structure of images, which often feature slowly varying regions, separated by sharp edges. It turns out that wavelet decompositions are very well adapted to such situations, in the sense that corresponding expansions may be extremely sparse, and that the significant wavelet coefficients generally group themselves into clusters, a property which is exploited in several coders $[23,25]$.

We shall be mainly concerned in this paper with the case of audiophonic signals. Transform coding strategies have been successfully applied to audio signals (see for example the various versions of MPEG audio coders). However, the structure of audio signals makes the signal encoding problem significanly different from the problem of image encoding, because of the possible superposition of different kinds of components: whereas different objects tend to occlude each other in images, they rather superimpose to each other in the case of (polyphonic) audio signals ${ }^{1}$. Since these components may have significantly different behaviors (for example, fastly varying transients, or slower "partials") it is difficult to derive a transform that can be simultaneously matched for all of them. It is the purpose of this paper to describe and analyze the main features of a new approach for modelling and encoding such "composite signals". The approach we propose is based on a signal model of the form

$$
\text { Tonals + transients + residual }
$$

and a simultaneous transform coding of the tonal and transient components, using different transforms [11] (we refer to [10] for a more detailed analysis.) The estimation and removal of transients from the signal plays the role of a "stationarization" of the signal; the estimation and removal of the tonal component plays a role similar to an estimation of peaks in the power spectrum. Hence, if the two components above

\footnotetext{
${ }^{1} \mathrm{~A}$ similar effect is also present in the case of transparent objects in images, but the effect is much less significant.
} 
are successfully estimated and removed from the signal, the residual should take the form of a stationary random signal with smooth power spectrum, and should be easy to describe by simple autoregressive models with short memory. This is the goal we shall follow in the present article.

It is important to notice that such a strategy automatically introduces redundancy into the representation, which results in extra cost in terms of the amount of data to encode. It is hoped that the adequacy of the models for the three considered components will result in significantly better compression rates for each of them, and a competitive global compression rate.

Such hybrid signal models are quite flexible, which opens interesting possibilities of signal processing directly in the transform domain. Among these, let us mention psychoacoustic processing such as masking (see e.g. [34] for a detailed presentation). Masking is the ability of human auditory system to ignore certain signals when they are received simultaneously with "close" signals with higher amplitude. Masking is actually used in modern audio coders: instead of minimizing the decoding error, the coded tries to make it "transparent" from a perceptive point of view (see for example [4, 22]). It is known that several types of masking (for which several models have been proposed) should be taken into account: temporal masking and frequency masking (including different phenomena for masking tonal and wide band signals). An hybrid representation for audio signals should allow one to implement different masking algorithms on different components, and therefore improve significantly coding schemes. Other examples of transform domain processing include several types of signal modifications, such as pitch transposition, speed modification,... Such modifications have been discussed in [31].

\section{AdAPted Representations FOR transform CODing}

In transform coding strategies, the transform step is equivalent to the expansion of the signal with respect to a suitably chosen basis. Among the most popular transforms for transform coding, the local cosine bases and wavelet bases have received particular attention. Local cosine bases have been shown to provide adequate approximations of Karhunen-Love bases for locally stationary signals, and wavelet bases (or the equivalent subband decompositions in the discrete case) have been extremely successful for coding signals exhibiting abrupt changes. We briefly sketch our notations here, before discussing the interest of each transform for specific kinds of signals.

2.1. Transform coding, adapted transforms, non-linear expansions. Transform coding [29, 30] amounts to perform a linear transformation prior to quantization and encoding. Such a linear transformation may be understood as a change of basis, or an expansion with respect to a specific basis of the considered space of signals. The goal of the transform is to "factor out" the redundancies present in the samples, and therefore to reduce the amount of significant coefficients. Transforms which achieve such goals are "adapted" to the signal class. A huge literature has been devoted to adapted transforms in the recent years. We refer to [16] and [30] and references therein for more details.

In the framework of transform coding schemes, when a large number of coefficients are below a given threshold (the accuracy of the encoder), encoding all the coefficients irrespective to their actual significance may become particularly unefficient, and it makes sense to encode only the significant coefficients. The resulting encoded signal is then a non linear approximation of the original signal, since the choice of the coefficients to be retained depends on the signal itself ${ }^{2}$. Obviously, such a strategy becomes really useful only when the necessary encoding of the "significance map" (i.e. the addresses of significant coefficients) may be achieved efficiently.

\footnotetext{
${ }^{2}$ Indeed, the corresponding expansion of the sum of two signals will not be the sum of the expansions of the signals.
} 
REMARK 1. One may also mention at this point the best basis strategies advocated in [33], which seek the transform optimizing the sparsity of the representation in a library of possible transforms. The resulting (signal dependent) basis is an adaptive basis, and has to be encoded.

For the present discussion, we shall limit ourselves to two special cases of transforms using bases adapted to specific signal features (and not adaptive bases), namely wavelet and local trigonometric bases. We shall also formulate the problems mainly in terms of expansion of analogic signals (i.e. we first describe the transform step in terms of choice of a basis in spaces of functions) before turning to a formulation adapted to discrete signals.

2.2. Wavelets and subband coding. We only sketch here the aspects which are relevant for our purpose, and limit ourselves to the simplest version of wavelet theory. More details on wavelet expansions (construction of filters, examples...) and generalizations (biorthogonal decompositions, wavelets on bounded domains,...) may be found for example in $[8,18]$.

Wavelets introduce themselves naturally in the framework of a multiresolution analysis (MRA for short). A MRA is associated with a pair of $L^{2}$ functions $\phi$ (scaling function) and $\psi$ (wavelet), satisfying the two-scale difference equations (or refinement equations)

$$
\phi(t)=\sqrt{2} \sum_{k} h_{k} \phi(2 t+k), \quad \psi(t)=\sqrt{2} \sum_{k} g_{k} \phi(2 t+k), \quad t \in \mathbb{R},
$$

where $h$ and $g$ are $\ell^{1}$ sequences (generally with finite support), whose discrete Fourier transforms $H$ and $G$ satisfy the "perfect reconstruction conditions":

$$
\begin{aligned}
|H(\omega)|^{2}+|G(\omega)|^{2} & =2, \\
H(\omega+\pi) \bar{H}(\omega)+G(\omega+\pi) \bar{G}(\omega) & =0 .
\end{aligned}
$$

With $\phi$ and $\psi$, associate the family of scaled and shifted copies $\phi_{j k}$ and $\psi_{j k}$, defined by

$$
\psi_{j k}(t)=2^{-j / 2} \psi\left(2^{-j} t-k\right), \quad \phi_{j k}(t)=2^{-j / 2} \phi\left(2^{-j} t-k\right) .
$$

Remarkably enough, given filters $h$ and $g$ satisfying equations (2), and additional technical conditions (the so-called Cohen-Lawton conditions, see for example [8]), there exist a corresponding MRA, such that the wavelets $\left\{\psi_{j k}, j, k \in \mathbb{Z}\right\}$ form an orthonormal basis of $L^{2}(\mathbb{R})$. Therefore, any $x \in L^{2}(\mathbb{R})$ may be decomposed as the scalar products

$$
x=\sum_{k} s_{j_{0} k} \phi_{j_{0} k}+\sum_{j \leq j_{0}} \sum_{k} d_{j k} \psi_{j k},
$$

where $j_{0}$ is a fixed reference scale index, and the coefficients are defined as

$$
s_{j k}=\left\langle x, \phi_{j k}\right\rangle, \quad \text { and } \quad d_{j k}=\left\langle x, \psi_{j k}\right\rangle .
$$

An important feature of wavelet expansions is the fact that the computation of the $s$ and $d$ coefficients may be done using a fast recursive algorithm, identical to the subband coding schemes [32]:

$$
\begin{gathered}
s_{j-1 k}=\sum_{\ell} \bar{h}_{2 k-\ell} s_{j \ell}, \quad \text { and } \quad d_{j-1 k}=\sum_{\ell} \bar{g}_{2 k-\ell} s_{j \ell} . \\
s_{j+1 \ell}=\sum_{k}\left(h_{2 k-\ell} s_{j k}+g_{2 k-\ell} d_{j k}\right) .
\end{gathered}
$$

The connection between wavelet expansions and subband coding allows the extension of wavelet transforms to the case of discrete signals. When only samples are available, the latter are generally identified 
with the scaling function coefficients $s$ at the finest scale, say, $j=0$ for simplicity (alternatives are discussed in [12]), and the corresponding wavelet coefficients at coarser scales $j>0$ are just computed ${ }^{3}$ using the relations (7).

REMARK 2. Unlike several authors (see for example [26]), we shall not consider in the present article the more general subband decompositions (wavelet packets, see [33]), yielding different partitions of the time-frequency plane. Such expansions may be taylored specially to match the critical frequency bands of human auditory system, and therefore become quite efficient in the audio coding context. However, since we shall be only interested in subband coding of transients, the choice of classical wavelets is a natural one. In addition, since our goal is to characterize transients, we limit ourselves to short filters, emphasizing time localization.

Signals with finite support may be treated in many different ways. The simplest one amounts to consider a signal defined on an interval, say $[0,1]$, as the restriction to $[0,1]$ of a 1-periodic function, and use corresponding wavelet expansions. The alternative consists in changing the construction rule for wavelets whose support intersect the boundaries of the interval, still preserving the very properties of wavelet bases (orthogonality, completeness, vanishing moments,...) We shall limit ourselves to the first solution, and refer to [7] for a discussion of wavelet bases on intervals.

A remarkable feature of wavelet bases lies in their behavior with respect to non linear approximation in a variety of functional spaces. The non linear approximation problem, which turn out to be very close to the algorithms which are used in practice, may be described as follows: given a signal $x$ and a fixed integer $N$, seek the best possible approximation of $x$ as a linear combination

$$
S_{N}=\sum_{n=0}^{N-1} c_{n} u_{i(n)}
$$

of $N$ basis vectors $u_{i(0)}, \ldots u_{i(N-1)}$ in a given basis: the best approximation is the one which minimizes a given norm of the residual:

$$
\min _{i}\left\|x-S_{N}\right\|
$$

For general choices of the basis (and the norm), the selection of the best non linear approximation turns out to be a difficult problem. However, in the case of wavelet bases, the situation becomes much simpler. For example, assuming that $x \in L^{p}([0,1])$, set $\delta_{j k}=2^{-(1 / p-1 / 2) j} d_{j k}$, pick the $N$ largest $\delta_{j k}$ coefficients and denote by $\Sigma_{N}$ the corresponding $N$ terms wavelet expansion. Then the striking result is that $\Sigma_{N}$ achieves the same asymptotic rate of convergence as $S_{N}$ : there exists a constant $C_{p}$ such that for all $x \in L^{p}([0,1]),\left\|x-\Sigma_{N}\right\|_{p} \leq C_{p} \inf \left\|x-S_{N}\right\|_{p}$. In addition, several families of functional spaces may be characterized by the speed of convergence of non linear wavelet approximations (we refer to [6, 13], ... for a detailed mathematical presentation). Among them, the Besov spaces have received a particular attention in the context of image modeling, since they provide good models for functions with controllable "density" of singularities of a given strength. For the same reasons, such models seem also adequate for describing transient signals.

\footnotetext{
${ }^{3}$ An alternative algorithm, based on an initial polyphase decomposition and a factorization into a series of simple lifting steps $[27,9]$ may also be used, resulting in more efficient implementations.
} 
2.3. Local trigonometric bases. It is well known that any square-integrable function on an interval may be represented by a Fourier series, or a cosine series, and that functions on the real line also possess such trigonometric expansions, obtained by first segmenting the real axis into blocks, and using classical trigonometric expansions within each block. More recently, smooth versions of such bases (avoiding brutal "block" segmentation) have been proposed (see [33] or [18] for a review). The construction (in the case of the real line; the adaptation to bounded domains is straightforward) goes as follows. Consider an increasing sequence of numbers $\left\{a_{k}, k \in \mathbb{Z}\right\}$, and further numbers $\eta_{k}$ such that

$$
a_{k}+\eta_{k}<a_{k+1}-\eta_{k+1}
$$

and consider a set of functions $w_{k}$ such that

$$
\left\{\begin{array}{l}
w_{k}(t)=1 \text { if } t \in\left[a_{k}+\eta_{k}, a_{k+1}-\eta_{k+1}\right], \\
w_{k}(t)=0 \text { if } t \notin\left[a_{k}-\eta_{k}, a_{k+1}+\eta_{k+1}\right] .
\end{array}\right.
$$

Set

$$
u_{k \nu}(t)=\sqrt{\frac{2}{\ell_{k}}} w_{k}(t) \cos \left[\frac{\pi}{\ell_{k}}\left(\nu+\frac{1}{2}\right)\left(t-a_{k}\right)\right],
$$

where $\ell_{k}=a_{k+1}-a_{k}$ is the length of the $k$-th interval. Notice that (9) becomes $\eta_{k}+\eta_{k+1} \leq \ell_{k}$. Then, if the windows $w_{k}$ satisfy the compatibility relations: $\forall \tau$ such that $0 \leq|\tau| \leq \eta_{k}$,

$$
\left\{\begin{array}{l}
w_{k-1}\left(a_{k}+\tau\right)=w_{k}\left(a_{k}-\tau\right) \\
w_{k}\left(a_{k}+\tau\right)^{2}+w_{k-1}\left(a_{k}+\tau\right)^{2}=1,
\end{array}\right.
$$

then the functions $\left\{u_{k \nu}, k, \nu \in \mathbb{Z}\right\}$ form an orthonormal basis of $L^{2}(\mathbb{R})$. Classical choices for the bell functions include sine functions (actually implemented in MPEG coders) and modified Kaiser-Bessel windows.

Again, the bases of $L^{2}$ spaces have their discrete counterparts. Discrete local cosine bases ${ }^{4}$ (based upon similar constructions) are described in [33] or [18], and have been used in several instances in signal coding, for example in the MPEG audio coders (see e.g. [4]).

REMARK 3. Again, we shall not use these expansions in full generality, and limit ourselves to expansions involving fixed size windows: $w_{k}(t)=w_{0}\left(t-a_{k}\right)$. The more general solution offers the possibility of better matching the tonal component of the signal, at the price of higher complexity.

The choice of local cosine bases is motivated by the will of describing tonal signals, i.e. signals which may be reasonably modeled as stationary signals within a given time frame, and may be in addition characterized by a few coefficients (which excludes "wide band" stationary or locally signals). Therefore, this opens the problem of non linear approximation by local cosine bases. This problem has been addressed by Gröchenig and Samarah [15], who have shown that the so-called modulation spaces introduced by Feichtinger in the eighties provide an appropriate setting for non linear approximation with local cosine bases. A function $x$ is well approximated by local cosine bases if and only if it belongs to a certain modulation space, i.e. if its continuous Gabor (or short time Fourier) transform $G_{x}$ (see [5] for notations) belongs to some $L^{p}\left(\mathbb{R}^{2}\right)$ space, with $1 \leq p<2$.

\footnotetext{
${ }^{4}$ Expansions with respect to these bases are sometimes called LOT (lapped orthogonal transforms) or - in the case of a sine window - MDCT (modulated discrete cosine transforms).
} 


\section{AN HYBRID MODEL, PROJECTIONS}

We are concerned in this paper with hybrid signal models that include components of different kinds. More specifically, we will limit our investigations to additive models of the form "Tonal + Transient + Noise". Such models have been considered in the literature in various contexts, for example for sound modeling and transformation (see for example [24]), or for encoding and compression (see [17] and [31] and references therein). However, the models developed in a signal encoding context are generally based upon a segmentation of the signal into its tonal and transient components. Transients and tonals are then encoded using different transforms.

We develop here an approach avoiding such a segmentation, by considering superpositions of transient, tonal and stochastic ${ }^{5}$ components:

$$
x(t)=x_{\text {ton }}(t)+x_{t r}(t)+x_{s}(t) .
$$

The main difficulty lies in the joint estimation of the three components. Ideally, a simultaneous estimation would be desirable, as it is difficult to predict in advance the respective bit rates to be assigned for each component. However such a procedure seems difficult to implement (except maybe using matching pursuit strategies [20], at the price of a very high computational cost), and we shall limit ourselves to individual estimates for the transients and the tonals, defining the stochastic part as a residual. The estimation of tonal and transient components follow similar lines, i.e. are in the spirit of transform coding schemes, using local cosine and wavelet bases respectively. However, in each case, only the largest coefficients in each time frame are retained. The algorithm therefore depends on a pair of threshold values, which are estimated adaptively within larger time frames. The choice of these threshold values is related to the step used in the quantization stage. For the sake of simplicity, we shall limit ourselves to (mid-tread) uniform quantization.

As an illustration of the potential benefits of such hybrid schemes, let us consider a simple example: we take a small portion of the MPEG test signal gspi35.16. We display in FiguRE 1 the original signal, MDCT approximations of the tonal part, and the residuals corresponding to two decompositions of the signal using MDCT only and MDCT and wavelets respectively. In the first case (second and third plots), we only used MDCT expansion: the signal was expanded with respect to a MDCT basis, and the 950 largest coefficients ( 1.45 percent of the total number of coefficients) were selected. One may clearly see in the non-tonal residual (plot 3) that the attack has been poorly captured by the MDCT basis, and is still present in the residual. The energy of the residual represents 9.97 percent of the total energy of the signal.

In the second case (plots 4 and 5), only the 850 largest MDCT coefficients (1.3 percent of the total number of coefficients) were selected, the corresponding tonal component was substracted, and the 100 top wavelet coefficients were selected to estimate the transient part. The corresponding residual (whose energy represents 6.58 percent of the total energy of the signal) is shown in the bottom plot. The residual has clearly a much smaller dynamical range in this case, and is much easier to model as a wide sense stationary process.

\subsection{Tonal component modeling and estimation.}

\footnotetext{
${ }^{5}$ The term "stochastic" refers to the terminology used in $[17,31]$, and to the model used to describe such a component; "residual" would be more appropriate.

${ }^{6}$ Available at http://www.tnt.uni-hannover.de/project/mpeg/audio/sqam/
} 

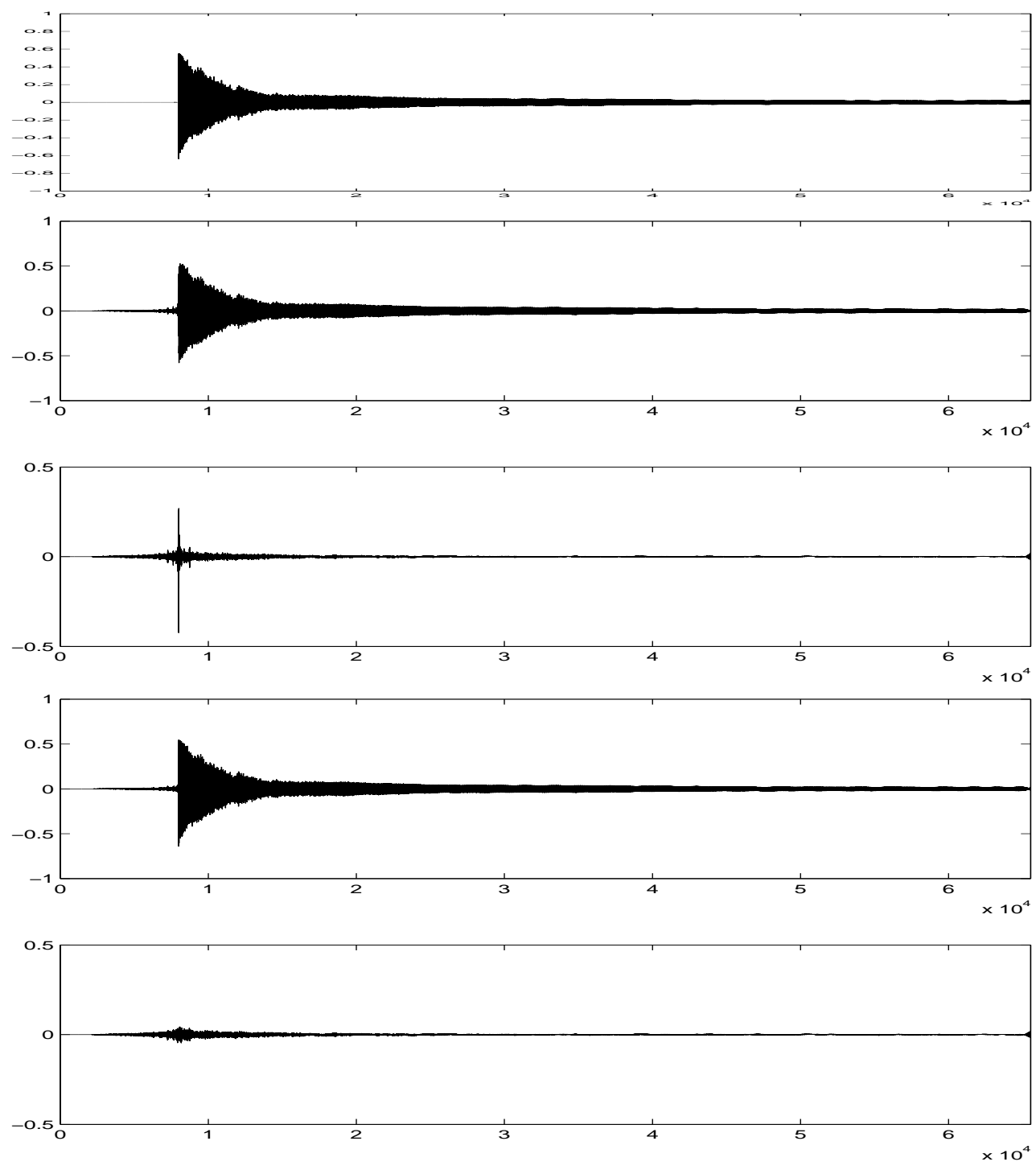

Figure 1. MDCT and MDCT+wavelet expansions: from top to bottom: a 65536 samples segment of the MPEG glockenspiel signal: approximation of a tonal part from 950 MDCT coefficients; corresponding residual; approximation of a tonal part from 850 MDCT coefficients and 100 wavelet coefficients; corresponding residual.

3.1.1. Tonals and local cosines. Estimation and modeling of "tonals" (or "sinusoidal partials") is a fairly classical topic in the sound and speech signal processing literature. Examples may be found for example in $[21,24]$, in different contexts. We stick here to transform coding schemes, and use expansions with respect to adapted bases. A "tonal" may be modeled as a locally stationary signal in the sense of [19], i.e. well (in other words, sparsely) represented in local cosine bases. The following example provides an illustration of the expected situation.

REMARK 4. Let us assume a model of the form

$$
\begin{aligned}
x_{\text {ton }}(t) & =A(t) Y(t), \\
7 & \text {, }
\end{aligned}
$$


where $A$ and $Y$ are uncorrelated second order random processes, such that the correlation function $C_{A}(t, s):=\mathbb{E}\{A(t) A(s)\}$ of $A$ is continuously differentiable and slowly varying, and $Y$ is wide sense stationary (hence $C_{Y}(t, s)=C_{Y}(t-s)$ ) and zero mean. Denote by $\mathcal{S}_{Y}$ the spectral density of $Y$. Given a local cosine basis $\left\{u_{k \nu}\right\}$ as defined in the previous section, we easily obtain the following approximate expression for the variance of the coefficients $\left\langle x_{t o n}, u_{k \nu}\right\rangle$ :

$$
\begin{aligned}
\mathbb{E}\left\{\left|\left\langle x_{\text {ton }}, u_{k \nu}\right\rangle\right|^{2}\right\} & =\int C_{A}(t, s) C_{Y}(t-s) u_{k \nu}(t) u_{k \nu}(s) d t d s \\
& =\frac{1}{2 \pi} C_{A}\left(c_{k}, c_{k}\right) \int\left|\widehat{u_{k \nu}}(\omega)\right|^{2} \mathcal{S}_{Y}(\omega) d \omega+r,
\end{aligned}
$$

where we have denoted by $c_{k}=\left(a_{k}+a_{k+1}\right) / 2$ the center of the window $w_{k}$. In the latter approximation, $r$ is a remainder term which depends on the gradient of $C_{A}$ :

$$
|r| \leq K \sup _{t, s \in \operatorname{supp}\left(w_{k}\right)}\left|\partial_{t} C_{A}(t, s)\right| .
$$

Hence, the more slowly varying $C_{A}$ (within the support of $w_{k}$ ), the better the approximation.

Taking into account the expression of $w_{k \nu}$, and assuming that the remainder $r$ can be neglected ${ }^{7}$, we finally obtain

$$
\mathbb{E}\left\{\left|\left\langle x_{\text {ton }}, w_{k \nu}\right\rangle\right|^{2}\right\} \approx \frac{1}{8 \pi} C_{A}\left(c_{k}, c_{k}\right) \int\left|\hat{w}_{k}\left(\omega-\pi(\nu+1 / 2) / \ell_{k}\right)+\hat{w}_{k}\left(\omega+\pi(\nu+1 / 2) / \ell_{k}\right)\right|^{2} \mathcal{S}_{Y}(\omega) d \omega .
$$

Since the windows $w_{k}$ are generally localized near the origin in the Fourier domain, the main contributions to such an expression are provided by the neighborhoods of $\omega= \pm \pi(\nu+1 / 2) / \ell_{k}$. Assuming that the power spectrum $\mathcal{S}_{Y}$ contains sharp peaks at frequencies $\omega_{1}, \omega_{2}, \ldots$, the coefficients $\left\langle x_{t o n}, u_{k \nu}\right\rangle$ such that $\nu+1 / 2 \approx \omega_{1} \ell_{k} / \pi, \ldots$ may be expected to be significantly large, so that they will be selected by an appropriate thresholding.

Similar results may be obtained using a slightly more sophisticated model allowing for frequency modulation: $x(t)=A(t) Y(t) e^{i \varphi(t)}$ with $A$ and $Y$ as before, and $\varphi$ is a (smooth) local phase function whose derivative $\varphi^{\prime}$ is slowly varying. The conclusion is similar, as long as the windows $w_{k}$ are such that the variations of $\varphi^{\prime}$ within each $w_{k}$ are small enough.

Summarizing, a tonal is expected to be adequately represented by (a small number of) significant local cosine coefficients.

3.1.2. Estimation of tonals. Following ideas developed by the Yale group [3], we then "model" the tonal component as the component of the input signal which is "best described" by local cosines. More precisely, let $\left\{u_{k \ell}\right\}$ denote an MDCT basis as given in (11), associated with a family of identical (sine) windows $w_{k}(t)=w\left(t-a_{k}\right)$ and an uniform segmentation of the time domain $\left(a_{k+1}=a_{k}+\ell\right.$ and $\eta_{k}=\eta$ for all $\left.k\right)$. The windows are chosen in such a way that the tonal components may reasonably be assumed stationary within the window. The windows' overlap is taken maximal (i.e. such that $\eta=\ell / 2$ ), so as to optimize frequency localization. A typical value for the window half-length $\ell$ is about 20 milliseconds (ie. 1024 coefficients at $44.1 \mathrm{kHz}$ sampling rate). Given the input signal $x$ we consider the MDCT coefficients

$$
\alpha_{k \ell}=\alpha_{\lambda}=\left\langle x, u_{k \ell}\right\rangle
$$

\footnotetext{
${ }^{7}$ Such an assumption implies restrictions on the choice of the windows $w_{k}$, in particular on their length $\ell_{k}$ : the windows $w_{k}$ have to be short enough to ensure that $C_{A}$ is slowly varying enough within $w_{k}$; on the other hand it has to be large enough to ensure a sufficient frequency resolution.
} 
where $\lambda=(k, \ell)$ denote a joint time-frequency index, and pick the following first (non linear) estimate of the tonal part

$$
x_{\text {ton }}^{0}=\sum_{n=0}^{N_{\text {ton }}-1} \alpha_{\lambda(n)} u_{\lambda(n)},
$$

where the coefficients $\alpha_{\lambda(n)}$ are the $N_{\text {ton }}$ coefficients $\alpha_{k \ell}$ whose modulus exceeds a given threshold $\tau_{\text {ton }}$. The determination of the threshold $\tau_{\text {ton }}$ (or the number of atoms $N_{t o n}$ ) is a crucial aspect of the algorithm. $\tau_{\text {ton }}$ must be small enough to capture most of the tonal information, but large enough to allow efficient compression. Since we work in the context of uniform quantization (mid-tread uniform quantizer, see [16]), the threshold $\tau_{\text {ton }}$ is also intimately related to the quantization intervals. More precisely, we take $\tau_{\text {ton }}$ in the vincinity of the quantization step (by analogy to the case of low-resolution transform coding strategies [18]).

Furthermore, in order to capture the dynamic (amplitude) variations of the signal, $\tau_{\text {ton }}$ must be adapted locally. In this work, we use the following strategy. The distribution of the $\alpha$ coefficients is estimated within a "large" time frame (consisting of several "small" time frames), and the critical value $z_{p}$ corresponding to a given $p$-value (i.e. such that $\mathbb{P}\left\{|\alpha| \geq z_{p}\right\}=p$ ) is estimated. The threshold $\tau_{\text {ton }}$ is a fixed multiple of the critical value:

$$
\tau_{\text {ton }}=\rho z_{p}
$$

Within a "small" time frame all coefficients $\alpha$ larger than the threshold are retained. Typical values for large and small frame lengths are about 100 milliseconds and 20 milliseconds respectively.

REMARK 5. The tonal component estimation may also be understood as a way of selecting locally the discrete component of the power spectrum. However, since our approach is based upon a transform followed by a thresholding (and not a simple "peak picking" as in sinusoidal coders), it may be necessary to correct for the overall behavior of the latter, which may vary by several orders of magnitude. Such a correction may be done by an appropriate normalization, as follows.

The selection of the significant coefficients $\alpha_{\lambda}$ may be performed on appropriately weighted coefficients (where the weighting depends on the frequency variable only): given a family of weights $\mathbf{w}_{0}, \mathbf{w}_{1}, \ldots$, consider the coefficients

$$
\tilde{\alpha}_{k \ell}=\mathbf{w}_{\ell} \alpha_{k \ell} .
$$

Denote by $\lambda(n), n=0,2, \ldots N_{t o n}-1$ the indices corresponding to the $N_{t o n}$ 1weighted coefficients $\tilde{\alpha}_{k \ell}$ whose modulus exceeds a given threshold $\tau_{\text {ton }}$ (and where we have set $\mathbf{w}_{k \ell}=\mathbf{w}_{k}$ ), and consider the approximation (14) obtained with this new set of coefficients Typical choices for the weighting functions are of the form

$$
\mathbf{w}_{\ell}=\frac{1}{\left(\nu_{0}+\nu(\ell)\right)^{\alpha}}
$$

where $\nu(\ell)$ is the value of the frequency corresponding to the frequency index $\ell$, and the constants $\nu_{0}$ and $\alpha$ are adjusted so as to tune the behavior at low frequencies (typically, frequencies much smaller than $\nu_{0}$ are not affected by the weighting) and the decay at high frequencies. The effect on such a normalization will appear clearly in Figure 5 below. 
3.1.3. Quantization and encoding. The encoding of the tonal component involves encoding of the addresses of retained coefficients, and quantization and encoding of their values. This information is sufficient for the decoder to reproduce the tonal component, according to equation (14).

Let us first focus on the quantization of the coefficients. Consider a uniform quantizer $Q$, and denote by $Q(\alpha)$ the quantized coefficients. The simplest approach consists in quantizing the $N_{\text {ton }}$ coefficients $\alpha_{\lambda(0)}, \ldots \alpha_{\lambda\left(N_{t o n}-1\right)}$, which yields the corresponding tonal component

$$
x_{\text {ton }}=\sum_{n=0}^{N_{\text {ton }}-1} Q\left(\alpha_{\lambda(n)}\right) u_{\lambda(n)},
$$

The quantized coefficients $Q\left(\alpha_{\lambda(0)}\right), \ldots Q\left(\alpha_{\lambda\left(N_{t o n}-1\right)}\right)$, are finally entropy coded.

The simple quantization above gives the same precision to all the frequency bands of the signal. However, it is known that different frequencies are not treated equally in the auditory system. In particular, high frequencies are much less audible than intermediate frequencies. In our case, a simple uniform quantization would lead us to spend a significant part of the allowed bit budget for coding a frequency domain which is poorly relevant from a perceptive point of view. Without going into sophisticated modeling of the auditory system (e.g. masking effects), it is nevertheless possible to take simple information into account by simple normalization of the coefficients, involving a modeling of the auditory perception threshold. We use the following expression, taken from [28]

$$
T_{q}(\nu) \approx 3.64 \nu^{-0.8}-6.5 e^{-0.6(\nu-3.3)^{2}}+0.001 \nu^{4},
$$

where the frequency $\nu$ is expressed in $\mathrm{kHz}$, and the threshold is in $\mathrm{dB}$. Setting

$$
A(\nu)=10^{T_{q}(\nu) / 10}
$$

we finally consider the following quantizer

$$
\tilde{Q}\left(\alpha_{k \ell}\right)=Q\left(\frac{\alpha_{k \ell}}{A(\nu(\ell))}\right)
$$

(where again $\nu(\ell)$ is the frequency associated with the index $\ell$ ) and the corresponding estimate of the tonal component,

$$
x_{\text {ton }}=\sum_{n=0}^{N_{\text {ton }}-1} \tilde{Q}\left(\alpha_{\lambda(n)}\right) u_{\lambda(n)} .
$$

The quantized coefficients $\tilde{Q}\left(\alpha_{\lambda(n)}\right)$ are finally entropy coded.

REMARK 6. It is interesting to notice the different roles of weightings.... blabla

3.2. Transient component modeling and estimation. After the estimation of a tonal component (21), the "non-tonal" signal is then defined as

$$
x_{n t}=x-x_{t o n}^{0},
$$

and has energy

$$
\left\|x_{n t}\right\|^{2}=\|x\|^{2}-\sum_{n=0}^{N_{t o n}-1}\left|\alpha_{\lambda(n)}\right|^{2} .
$$


3.2.1. Transients. According to the discussion of Section 2.2, transients are components of the signal which exhibit fast variations, and are therefore well represented in a wavelet basis. This means that transients should be characterized by a small number of large wavelet coefficients. An expansion of the signal (with the tonal component removed) with respect to a wavelet basis (using a wavelet $\psi$ with sharp time localization, i.e. filters with short impulse response) followed by a suitable thresholding is therefore expected to provide an estimate for the transient component.

In order to reduce boundary effects (which would manifest themselves as "transients"), the wavelet coefficients corresponding to a given time frame are computed a periodized wavelet transform on a larger (say, 3 times bigger) time frame. Within such a framework, it is possible to adjust the depth (i.e. the maximum number of scales) of the expansion to the frame length and the filter length so as to keep perfect reconstruction.

3.2.2. Transient estimation. The procedure for transient estimation (in its simplest form, see Section 4 below for the "structured" version) is very similar to the previous one. Starting from the estimated non-tonal signal within a "small" time frame, we seek the part corresponding to the largest wavelet coefficients. Introducing a joint "time-scale" index $\mu=(j, k)$ and a family of scale-dependent weights $\mathbf{w}_{0}, \mathbf{w}_{1}, \ldots$ (introduced in order to take into account some overall behavior of the coefficients accross scales, as in Remark 5), set

$$
\beta_{j k}=\beta_{\mu}=\left\langle x_{n t}, \psi_{j k}\right\rangle, \quad \text { and } \quad \tilde{\beta}_{j k}=\tilde{\beta}_{\mu}=\mathbf{w}_{j} \beta_{j k},
$$

and define

$$
x_{t r}^{0}=\sum_{m=0}^{N_{t r}-1} \beta_{\mu(m)} \psi_{\mu(m)},
$$

where the sum is taken over the set if indices $\mu(m)$ corresponding to the $N_{t r}$ largest coefficients $\tilde{\beta}_{j k}$, whose modulus exceeds a given threshold $\tau_{t r}$.

The threshold $\tau_{t r}$ may be determined along lines similar to the estimation of $\tau_{t o n}$ in (15): the empirical distribution of the $\beta$ coefficients is estimated within a "large" window, and the threshold $\tau_{t r}$ is set to a fixed multiple of a given percentile.

3.2.3. Quantization and encoding. The resulting coefficients are (uniformly) quantized, and entropy coded. Denoting by $Q_{t r}$ the quantizer used for the $\alpha$ coefficients,

$$
x_{t r}=\sum_{m=0}^{N_{t r}-1} Q\left(\beta_{\mu(m)}\right) \psi_{\mu(m)},
$$

3.3. Modeling the residual. The residual component of the signal is defined by

$$
x_{r e s}=x_{n t}-x_{t r},
$$

and has energy

$$
\left\|x_{r e s}\right\|^{2}=\|x\|^{2}-\sum_{n=0}^{N_{t o n}-1}\left|\alpha_{\lambda(n)}\right|^{2}-\sum_{n=0}^{N_{t r}-1}\left|\beta_{\mu(n)}\right|^{2} .
$$

If the estimation of the transient and the tonal parts are successful, the residual should behave as a wide band (locally) stationary signal, and is modeled as such. More precisely, $x_{r e s}$ is modeled using an autoregressive model of fixed length (a typical value for the filter length is 20 samples). The model parameters are estimated using a standard Levinson algorithm. This procedure is essentially the same as 


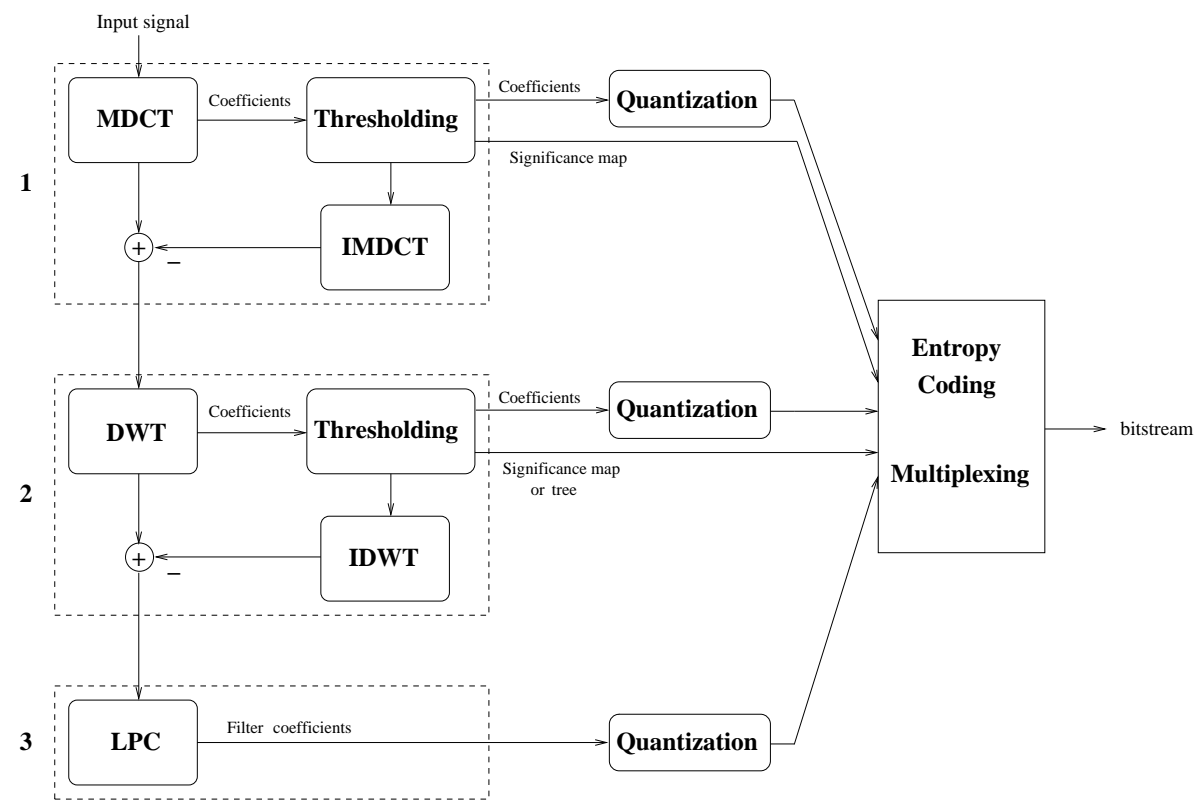

Figure 2. The prototype hybrid coder; MDCT and IMDCT stand for direct and inverse modulated cosine transforms respectively; DWT and IDWT stand for direct and inverse discrete wavelet transform respectively; LPC stands for linear predictive coding.

the one used in LPC coders used for speech coding. The filter coefficients are quantized and encoded. In the present stage, we use uniform quantization with 16 bits per coefficient.

In the decoding stage, the residual is simulated as a stationary stochastic process, using the AR model with the prescribed coefficients, using a pseudo random number generator.

3.4. A simple hybrid encoding scheme. We give here the main steps of a simple scheme implementing the above approach (see also Figure 2), and exhibit corresponding numerical results for illustration.

(1) Tonal component estimation:

- Expand the signal $x$ with respect to a local cosine basis.

- Pick the most significant coefficients and form the tonal part $x_{\text {ton }}$ in (18).

- Quantize the significant coefficients (uniform quantization).

- Encode the significance map (run length coding).

- Substract the tonal part.

(2) Transient component estimation:

- Expand the non-tonal signal $x_{n t}$ with respect to a wavelet basis.

- Pick the most significant coefficients, and form the transient part $x_{t r}$.

- Quantize the significant coefficients (uniform quantization).

- Encode the significance map (run length coding).

- Substract the transient part.

(3) Residual estimation:

- Fit an autoregressive model of given order to $x_{\text {res }}$.

- Quantize the filter coefficients.

(4) Entropy coding, multiplexing 
The interest of such hybrid representations is best illustrated by audio signals containing both tonals and transients. We display here results obtained on a small segment of the glockenspiel gspi35.2, (65536 samples, $44.1 \mathrm{kHz}, 16 \mathrm{bit} / \mathrm{sample})$ taken from the MPEG sound examples ${ }^{8}$. The signal is displayed in FIGURE 3, together with its spectrogram -which indeed exhibits tonals and transients.

The hybrid decomposition (without quantization) is shown in Figure 4. We used windows of length 2048 (about 45 milliseconds), and the tonal estimate corresponds to 606 (weighted) MDCT coefficients $(0.92 \%)$. The weighting parameters were set to $\alpha=2$, and $\nu_{0} \approx 3000 \mathrm{~Hz}$. The tonal estimate still exhibits transitions which appear sharp on the plot, but turn out to be "slower" in a closer examination; it also features an expected "pre-echo" effect. As may be seen, the removal of the tonal estimate yields sharp attacks, together with a small "noise-like" component. Listening the corresponding sounds ${ }^{9}$ shows a satisfactory separation between what one would intuitively call attacks and tonals (even though the nontonal part seems to contain tonal information, which appears difficult to avoid.)

The weighting (see REMARK 5) appears to play a significant role in the resolution of the tonal component. This is best illustrated by FIGURE 5, where we display the power spectra of the different components using unweighted and weighted tonal estimates. The power spectra were estimated using a simple periodogram, without any smoothing since we want here to emphasize the behavior of the discrete part (the peaks) of the spectrum. The second and third plots (starting from top) show that the unweighted MDCT procedure fails at picking all the peaks in the power spectrum: the high frequency components are not well resolved in the tonal estimate, and are still significantly present in the non tonal one. Such an effect is clearly corrected by the weighting. In the weighted tonal estimate, all the peaks are satisfactorily resolved.

3.5. Alternate projections. The approach above may be criticized in several respects. A potential shortcoming of the "two-steps" estimation of the tonal and transient components is that the estimation of tonals is biased by the presence of transients and vice versa. To avoid such drawbacks, an alternative strategy amounts to use alternate projections, as suggested in [3]: first estimate a tonal component using MDCT, with a large value of the threshold $\tau_{\text {ton }}$ (or a small value of $N_{\text {ton }}$ ), and substract it from the signal. One then obtains a very small number of "tonal atoms", which are substrated from the signal. Then estimate a transient component, still with a large $\tau_{t r}$, which results in a small number of wavelets. Then, iterate the procedure, until the residual may be satisfactorily encoded using LPC. Refering to Figure 2, steps $\mathbf{1}$ and $\mathbf{2}$ are iterated several times before moving to step $\mathbf{3}$.

Such an approach is very much in the spirit of additive models and matching pursuit (see [14]). One expects a priori more precise estimates for the tonals and transients, at the price of an increased computational burden. Notice that such a procedure only modifies the estimation part, the decoding stage is not affected.

However, in our experiments, implementing such a two steps estimation procedure does not seem to improve significantly the results in terms of the parsimony of the representation. Likewise, matching pursuit strategies, while being much more computer intensive, do not seem to yield much sparser expansions. This seems to indicate that the considered bases are sufficiently different to separate transient and tonal components without iterative strategies. In addition, a complete implementation of such methods requires updating the MDCT and wavelet coefficients at each stage, which yields a large computational cost.

In this respect, it is worth mentioning the recent work [2] which addresses the problem of blind source separation from a single captor, a problem similar to the one we address here. The authors propose a

\footnotetext{
${ }^{8}$ Available at http://www.tnt.uni-hannover.de/project/mpeg/audio/sqam/

${ }^{9}$ Corresponding wav files are available at ???????????
} 

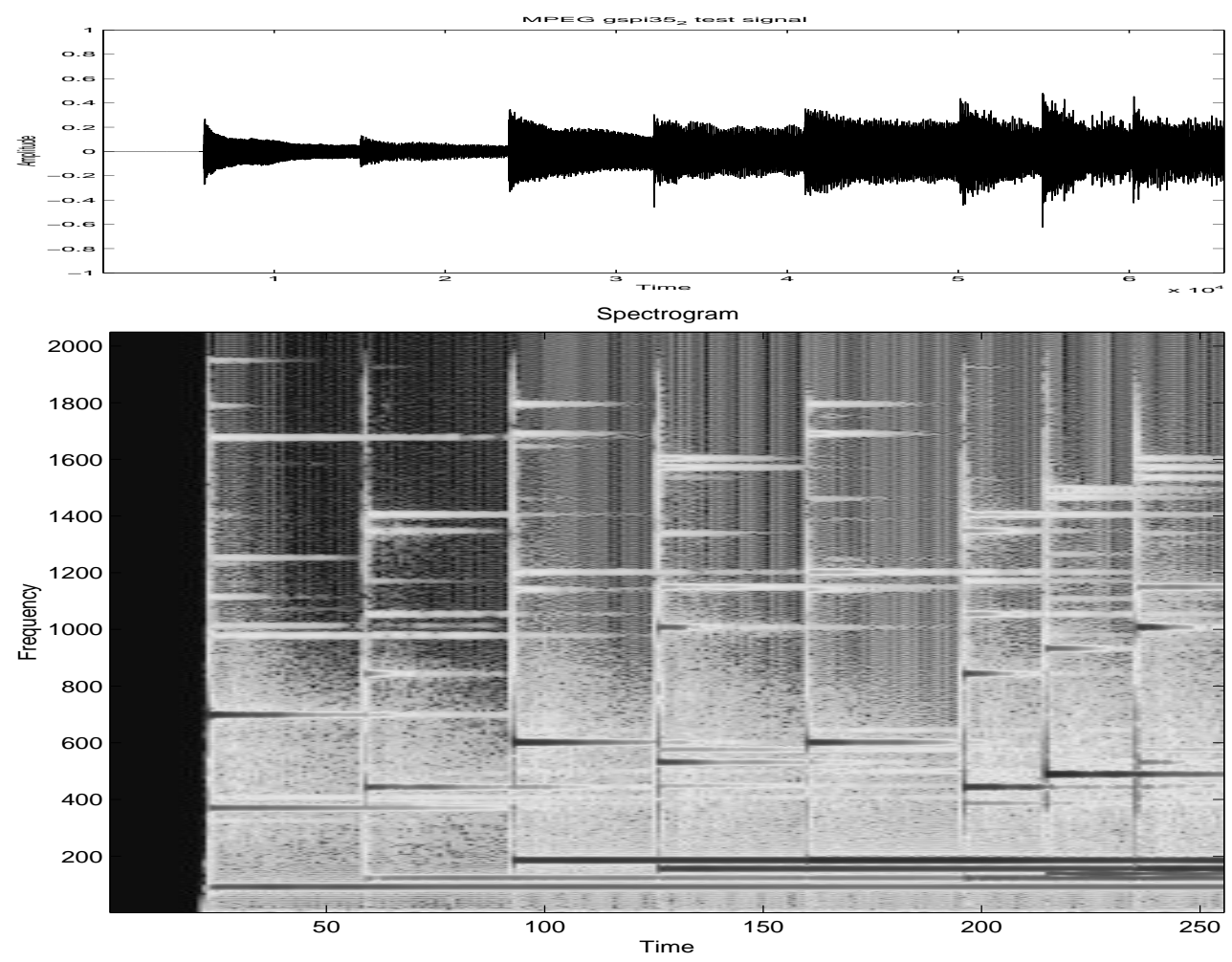

FigURE 3. MPEG test signal (Glockenspiel) and its spectrogram.
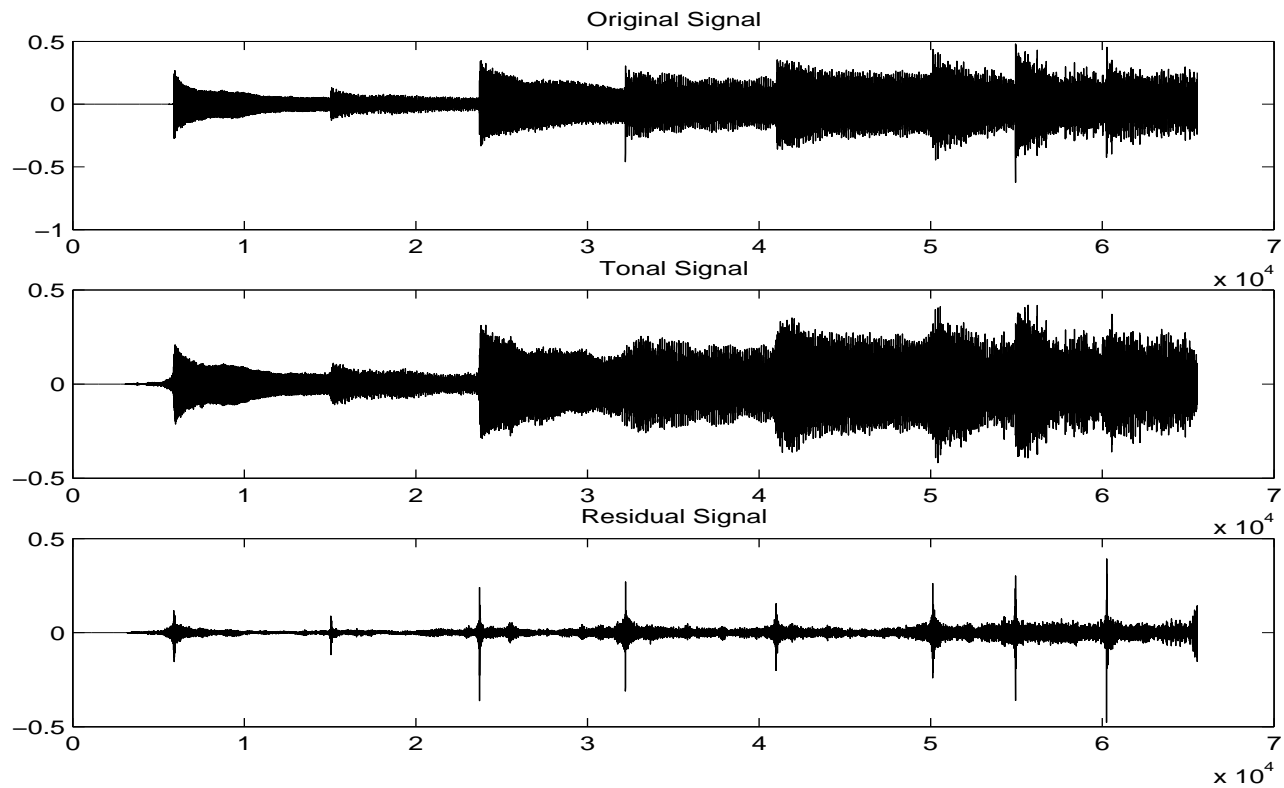

Figure 4. Hybrid decomposition MPEG gspi35.2 test signal. From top to bottom: original signal, tonal and non tonal parts, parts, estimated using weighted MDCT. 

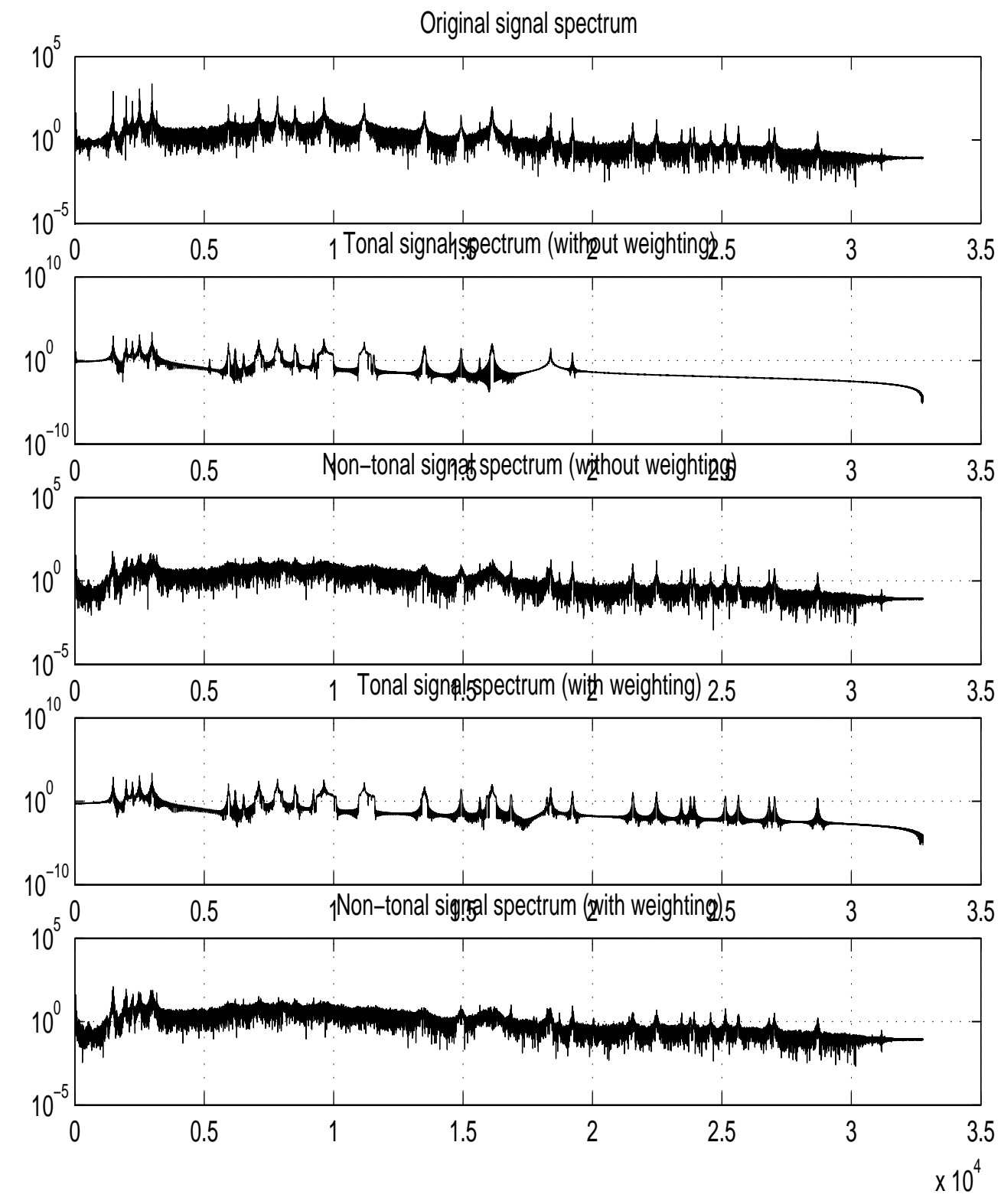

Figure 5. Power spectra for the MPEG gspi35.2 test signal. From top to bottom: original signal, tonal and non tonal parts, estimated without weighting the MDCT coefficients, and tonal and non tonal parts, estimated with weighting the MDCT coefficients.

criterion for testing the discriminating power of different bases, and it would be interesting to study this criterion in the case of the bases considered here.

\section{Structured Expansions}

4.1. The interest of structured expansions. As stressed already, transform coding based on non linear expansions opens the problem of encoding the addresses of significant coefficients. In the absence of additional information, the cost of significance map encoding is one bit per sample if a single basis is used, and two bits per sample if two different bases are used as in our case. If the representation of the 

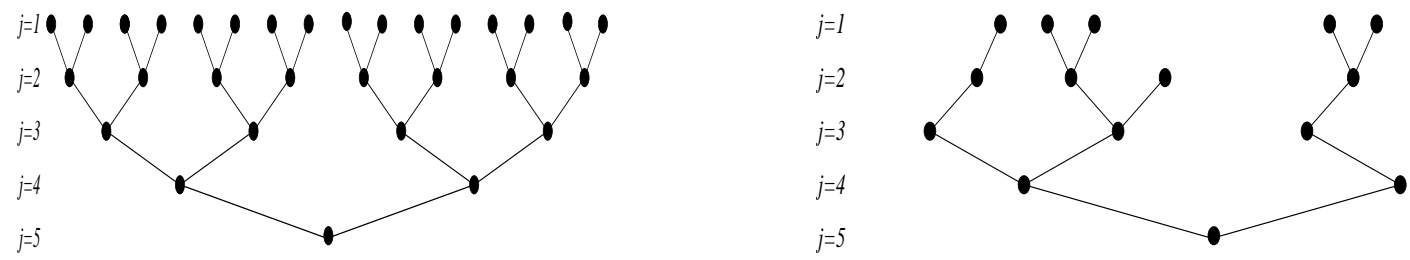

FiguRE 6. Wavelet coefficients grid, and a subtree of significant coefficients.

signal is sparse enough, direct encoding of addresses may nevertheless become efficient: for example, if in a given time frame of length say $L=2^{J}$ samples, only $N$ coefficients out of $L$ are significant, encoding their address results in a cost of $N J$ bits, which may be much less than $L$ (using typical values, $N=50$ significant coefficients out of $L=1024$ results in a cost of approximately half a bit per coefficient for the significance map).

However, this cost may be significantly reduced if the significant coefficients are "structured", i.e. if they are not distributed arbitrarily. When the significant coefficients are structured, the structure information may be used to reduce the address encoding cost. A simple example is provided by runlength coding of significance maps: if significant and unsignificant coefficients are not distributed in an homogenous way, i.e. if they have a tendancy to group into clusters of significant or unsignificant coefficients, it becomes "cheaper" to encode the lengths of the clusters (using entropy coding if necessary) rather than the significance map itself.

4.2. Trees of significant wavelet coefficients. We shall discuss here another kind of (deterministic) structure, and limit our discussion to the coding of transient components (similar ideas might be developed in the case of the tonal component, along lines similar to the sinusoidal models discussed in [21], or using adapted matching pursuits [14], but we shall not follow those lines here). Our starting point is the fact that transients do not only manifest themselves by large wavelet coefficients (in particular at small scales), but rather by clusters of significant wavelet coefficients [10].

4.2.1. A tree model for transients. Because of the natural dyadic grid structure of the wavelet coefficients ${ }^{10}$ (see FIG. 6), the latter often take the structure of a dyadic tree, which suggests to use dyadic trees of wavelet coefficients as a model for transients. In a structural model for transients, a transient is defined by a dyadic tree of significant wavelet coefficients, connected in the following sense: a wavelet coefficient $\alpha_{j+1,2 k}$ or $\alpha_{j+1,2 k+1}$ can be considered significant only if the parent coefficient $\alpha_{j, k}$ is significant. Such an approach is very much in the spirit of the the image coders based upon trees of (significant or unsignificant) wavelet coefficients, for example the EZW [25] of the SPIHT [23] algorithms - however in our case we only consider trees whose roots are at the largest scale.

Such a connected tree of wavelet coefficients is considered relevant if for all connected branches $\mathcal{B}$, the corresponding wavelet coefficients are significant in some average. This is mathematically expressed by the fact that the following modulus of regularity

$$
\kappa_{q, s}[\mathcal{B}]=\frac{1}{|\mathcal{B}|} \sum_{(j, k) \in \mathcal{B}} 2^{j s}\left|\alpha_{j, k}\right|^{q},
$$

\footnotetext{
${ }^{10}$ Assuming for the sake of simplicity that the function $\psi$ is centered at the origin $t=0$, then the wavelet $\psi_{j k}$ is centered at $t=k 2^{j}$, which yields a dyadic grid.
} 
exceeds a given maximum value (here, $|\mathcal{B}|$ denotes the length of the branch $\mathcal{B}$ ). A branch $\mathcal{B}$ is called a full length branch if its leaves always correspond to the finest considered scales. A tree is a complete tree if all its branches are full length branches.

The choice of assigning a cost to the branches of the trees (and not to the trees themselves) is motivated by the will of assigning a local feature to a transient: the leaves of the branches are naturally associated with samples in the signals.

The constants $s, q$ characterize the considered type of transients, in the sense that they weight coefficients corresponding to different scales (here, $2^{j s}$ plays the same role as the weight $\mathbf{w}_{j}$ in section 3.2.) For example, large (positive) values of $s$ emphasize large scales, and favor trees with short branches, whereas smaller values favor longer branches. A choice of $s$ therefore represents an "a priori" model for the transients to be considered. It can be mentionned that higher-complexity models with more general scale-dependent weights can be considered. The choice of $q$ also influences the type of transients to be estimated, in the sense that small values of $q$ enforce sparsity.

4.2.2. Tree estimation. In practice, the tree (i.e. the transient associated with the tree) has to be estimated from the signal, and encoded. For the estimation of transients, we use a dual "top-down" search algorithm: first a tree containing full-length branches only (ie. maximum length) is selected, and secondly these branches are pruned from unsignificant sub-branches.

Starting from a leave $\ell$ of the complete tree, consider the branch of all its ancestors, denoted by $\mathcal{D}_{\ell}$, and form the following quantity:

$$
\kappa_{q, s}[\ell]=\sum_{(j, k) \in \mathcal{D}_{\ell}} 2^{j s}\left|\alpha_{j, k}\right|^{q}
$$

where $s, q$ characterize the type of transients which are to be retained. In the numerical examples given below, we limit ourselves to the simplest choices $s=0$ and $q=1$ or 2 . The selection of full branches is done by retaining the leaves of the tree $\ell$ such that $\kappa_{q, s}[\ell]$ exceeds a threshold value $\tilde{\kappa}$.

In a second top-down pass, sub-branches of insignificant coefficients are pruned down: starting from scale $j=1$, one prunes down leaves that are insignificant with respect to a certain (fixed) threshold $\rho$. Note that only leaves are considered in this stage, which ensures that after pruning the trees remains connected. A good choice for $\rho$ in the case $s=0$ and $q=1$ is $\rho=\tilde{\kappa} / J$, which represents the mean of the absolute value of the coefficients on a branch that has just been selected during the first stage.

In practice, this second pass often reduces the number of coefficients by a very significant amount (typically by a factor 2 to 3 ), as small-scales coefficients have usually very small amplitudes. All the wavelet coefficients belonging to the retained tree are then quantized and encoded. The corresponding "significance tree" has also to be encoded, which requires 1 bit to encode the significance of the root, 2 bits per node at scales $j=2 \ldots J$ (to specify the significance of the children of the node), and nothing for leaves at the smallest scale $j=1$ (they can't have significant children). For example, the tree exhibited in Figure 6 (right) requires 21 bits for encoding the significance tree (15 coefficients).

REMARK 7. Again, the selection of the threshold $\tilde{\kappa}$ is a crucial ingredient. In order to capture the whole dynamics of the signal, a good alternative is to adapt it locally to the signal. The value $\tilde{\kappa}[\ell]$ has to be estimated within a time frame larger than the time frame defined by the complete tree. This may be done using a comparison of the $\kappa$ function with a smoothed version $\tilde{\kappa}$. Given such a smoothed version, 
consider all the values $\ell$ such that

$$
\frac{|\kappa[\ell]-\tilde{\kappa}[\ell]|}{\kappa[\ell]} \geq p
$$

where $p$ is a reference percentile.

REMARK 8. There exist many alternatives to the method presented here for tree estimation. Let us mention for the record the approach based upon hidden Markov trees of significant wavelet coefficients developed in [11] following [1], which model the signal with a mixture random model consisting of "transient" wavelet coefficients and "non transient" coefficients.

Besides being well adapted to the description of transient signals, we have seen in this this section that tree structures are also quite efficient in terms of coding, as one can easily construct strategies that require a maximum of 2 bits per retained coefficient for encoding the significance map of a binary tree. Therefore, if the number of retained coefficients is small enough (which is one of the objectives of transform coding strategies), the cost of significance map encoding is expected to be quite low.

However, the underlying assumption that significant coefficients have a significant parent is sometimes not verified, which results in cases where we have to encode unsignificant coefficients. Statistics based on a wide variety of sounds show that the overall gain is usually very much in favour of this stuctured approach.

4.3. Results, parameter estimation. In terms of coding gains, the use of tree structures can be seen on fig.

\section{Discussion AND PERSPECTIVES}

Besides the aformentionned application to audio coding, hybrid schmes as presented in this paper are a good framework for numerous applications in the field of audio analysis and synthesis. Let us review three of these, namely masking modelling, audio effects and high-level audio representations.

- Masking modelling One of the shortcomings of subband-based audio coding schemes, like those derived from the MPEG family, is that they rely heavily on the psychoacoustic model. Masking models constructed from the spectrum and based on psychoacoustic models, tell us what quantization noise is tolerable in each subband, ie. what is the minimum number of bits that we are allowed to use in the quantization stage in order to ensure "transparency". Because all coefficients in a given subband are considered and subject to quantization (on a small or even zero number of bits, hence the data reduction), the estimation of a correct making curve is a key point. One main objection is that the validity of the models for signals exhibiting a large number of spectral components is highly questionnable, as it is for non-stationary sources (for instance the only value of the spectrum my let us misinterpret a sharp but well-defined transient as some wide-band noise).

On the other hand, transform coding (where the large majority of coefficients is set to zero and only the coefficients that are selected as significant are quantized), one is less sensitive to the design of the (non-uniform) quantizer. Nonetheless, psychoacoustic principles can still be applied to improve the perceptual quality of the coding scheme. In this respects, an efficient separation in tonal, transient and stochastic components is a good framework for an enhanced psychoacoustic modelling. The tonal and stochastic contributions in each frequency channel are a natural estimate for the tonality index that appears in the computation of thresholds in the 
MPEG psychoacoustic model. Moreover, the information on sharp transients can be used to implement explicitely some temporal masking.

Is is believed that psychoacoustic modelling can be significantly improved by the use of redundant (overcomplete) representations, in the same way as redundancy itself plays an important role in the neuro-cognitive processes of human perception [34].

- Signal modifications Following the same lines, we can also discuss the importance of separating the tonal, transient and stochastic components of a soundfile for a wide range of sound effects. Amongst them, let us focus specifically of time stretching without pitch modification - or its dual problem (up to a global resampling) pitch shifting without time modifications. This problem ill-posed from a strict mathematical point of view - is one of the most difficult to implement in order to handle the largest variety of sounds.

Most popular methods are based on the now classical phase vocoder (frequency-domain methods), or waveform duplication (time-domain methods). However, when sharp transients are present in the signal, none of these approches gives satisfactory results, as frequency methods tends to smear these transients, whereas time-domain methods would duplicate them. Indeed, a perceptually good algorithm needs to leave the transients unchanged. Our method can therefore be used as a transient detection scheme, and a segmentation can be performed between steady-state (processed using standard schemes) and transient regions (left unchanged). Spectral enveloppe modelling can also be used in the tonal and stochastic parts in order to preserve formantic structures.

- High-level signal representations Another promising aspect in this framework of representations based on an explicit model of the structure of musical signals is that it can lead to higher-level representations, such as single notes (or more generally objets sonores). The different classes of such sound objects could be parametrised with features issued from our scheme. For instance a single organ note could be described as an attack (made of transients) and a sustained part (composite with a tonal part - harmonic - and stochastic components - the typical turbulence noise associted with the reed-jet interaction). This is very much in the spirit of object-oriented coders, such as MPEG-4. However, the transcription algorithms (ie. in this case the passage from soundfiles to "object" files, typically a pattern recognition algorithm) will obviously become of extreme complexity in the most general polyphonic multi-instrumental case.

\section{Conclusion}

In this paper we have discussed some advantages of overcomplete representations for audio signals. Amongst representation spaces, a union of local cosine and dyadic wavelets have proved good separation properties for structural features of audio signals, their tonal part and transient part respectively. The separation of these two layers is improved by the use of structured representations, that also greatly reduce the cost of encoding significance maps (these structures can be seen as a way to enforce constraints that have been released by overcompleteness). The residual of these models can generally be modelled without perceivable difference as stochastic processes with slowly-varying parameters.

It is imporant to note that the algorithms presented here are all based of "fast" numerical schemes and do not require any prior training. Furthermore, their small number of parameters ensure that they can be applied with comparable results to most types of audio materials.

It is believed that these schemes could have numerous applications, particularly for the coding of "highquality" sounds (like those issued from Super Audio CD, or DVD-Audio sources), for whom traditionnal 
coders are clearly sub-optimal. One of the main advantages of this method is that they can retain a very high sound quality even at high compression ratios without the need of complicated psychoacoustic models.

Many open questions have been mentionned in the body of the article. Future research will primarily focus on the choice of time-varying parameters, and on the construction of structures across adjacent windows for the selection and encoding of tonal components.

\section{REFERENCES}

[1] R. Baraniuk. Optimal tree approximation using wavelets. In A. J. Aldroubi and M. Unser, editors, Wavelet Applications in Signal Processing, volume VII, pages 196-207. SPIE, 1999.

[2] L. Benaroya, R. Gribonval, and F. Bimbot. Représentations parcimonieuses pour la séparation de sources avec un seul capteur. In Flandrin P., editor, GRETSI'01, 1991.

[3] J. Berger, R. Coifman, and M. Goldberg. Removing noise from music using local trigonometric bases and wavelet packets. J. Audio Eng. Soc., 42(10):808-818, 1994.

[4] K. Brandenburg. MP3 and AAC explained. In Proc. of AES 17th international conference on High Quality Audio Coding (Florence), september 1999.

[5] R. Carmona, W.L. Hwang, and B. Torrésani. Practical Time-Frequency Analysis: continuous wavelet and Gabor transforms, with an implementation in S, volume 9 of Wavelet Analysis and its Applications. Academic Press, San Diego, 1998.

[6] A. Cohen, W. Dahmen, I. Daubechies, and R DeVore. Tree approximation and optimal encoding. IGPM Report, RWTH Aachen, sept 1999.

[7] A. Cohen, I. Daubechies, B. Jawerth, and P. Vial. Multiresolution analysis, wavelets and fast algorithms on an interval. Comptes Rendus Acad. Sc. Paris, 1992.

[8] I. Daubechies. Ten lectures on wavelets. SIAM, Philadelphia, PA, 1992.

[9] I. Daubechies and W. Sweldens. Factoring wavelet transforms into lifting steps. J. Fourier Anal. Appl., 4(3):245-267, 1998.

[10] L. Daudet. Représentations structurelles de signaux audiophoniques. Méthodes hybrides pour des applications à la compression. PhD thesis, Université de Provence, 2000.

[11] L. Daudet, S. Molla, and B. Torrésani. Transient modeling and encoding using trees of wavelet coefficients. In GRETSI'01, Toulouse, September 2001.

[12] B. Delyon and A. Juditsky. On the computation of wavelet coefficients. Journal of Approximation Theory, 88(1):47-79, 1997.

[13] R.A. DeVore, B. Jawerth, and B.J. Lucier. Image compression through wavelet transform coding. IEEE Trans. on Information Theory, 2:719-746, 1992.

[14] R. Gribonval. Approximations non-linéaires pour l'analyse des signaux sonores. PhD thesis, Université de Paris IX Dauphine, 1999.

[15] K. Grochenig and S. Samarah. Non-linear approximation with local fourier bases. Constr. Approx., 16:317-332, 2000.

[16] N. S. Jayant and P. Noll. Digital coding of waveforms. Prentice-Hall, 1984.

[17] S. Levine. Audio Representations for Data Compression and Compressed Domain Processing. PhD thesis, Stanford University, 1998.

[18] S. Mallat. A wavelet tour on signal processing. Academic Press, 1998.

[19] S. Mallat, G. Papanicolaou, and Z. Zhang. Adaptive covariance estimation of locally stationary processes. Ann. Stat., 26(1):1-47, 1998.

[20] S. Mallat and Z. Zhang. Matching pursuits with time-frequency dictionaries. IEEE Transactions on Signal Processing, 41:3397-3415, 1993.

[21] R.J. McAulay and Th.F. Quatieri. Speech analysis/synthesis based on a sinusoidal representation. IEEE Trans. on Acoust., Speech and Signal Proc., 34:744-754, 1986.

[22] T. Painter and A. Spanias. Perceptual coding of digital audio. Proc. IEEE, 88(4), april 2000.

[23] A. Said and W. A. Pearlman. A new, fast, and efficient image codec based on set partitionning in hierarchical trees. IEEE Trans. on Circ. and Syst. for Video Tech, 6(3):243-250, 1996. 
[24] X. Serra. A System for Sound Analysis/Transformation/Synthesis based on a Deterministic plus Stochastic Decomposition. PhD thesis, Stanford University, 1989.

[25] J. M. Shapiro. Embedded image coding using zerotrees of wavelet coefficients. IEEE Trans. Signal Processing, 41(12):3445-3462, 1993.

[26] D. Sinha and A. Tewfik. Low bit rate transparent audio compression using adapted wavelets. IEEE Trans. Signal Processing, 41(12):3463-3479, 1993.

[27] W. Sweldens. The lifting scheme: A construction of second generation wavelets. SIAM J. Math. Anal., 29(2):511-546, 1997.

[28] E. Terhardt. Calculating virtual pitch. Hearing Research, 1:155-182, 1979.

[29] P.P. Vaidyanathan. Multirate systems and filter banks. Prentice Hall, Englewood Cliffs, NJ, USA, 1993.

[30] P.P. Vaidyanathan and S. Akkrarakaran. A review of the theory and applications of optimal subband and transform coders. Appl. and Comp. Harm. Anal., 10:254-289, 2001.

[31] T. Verma, S. Levine, and T. Meng. Transient modeling synthesis: a flexible analysis/synthesis tool for transient signals. In Proc. of the International Computer Music Conference, Greece, 1997.

[32] M. Vetterli and J. Kovacevic. Wavelets and subband coding. Prentice Hall, Englewood Cliffs, NJ, USA, 1995.

[33] M. V. Wickerhauser. Adapted Wavelet Analysis from Theory to Software. AK Peters, Boston, MA, USA, 1994.

[34] E. Zwicker and H. Fastl. Psychoacoustics, facts and models. Springer Verlag, 1990.

Addresses:

L. DAUDET

Dept. of Electronic Engineering, Queen Mary, University of London, Mile End Road, London E1 4NS, UK.

B. TORRÉSANI

LATP,

CMI, Université de Provence, 39 rue Joliot-Curie, 13453 Marseille Cedex 09, F. 